\title{
EXPERIENCIAS
}

\section{ASESORAMIENTO PSICOPEDAGÓGICO Y ORIENTACIÓN VOCACIONAL EN UN CASO DE DISTROFIA MUSCULAR DUCHENNE}

\section{GUIDANCE AND VOCATIONAL COUNSELTING AIMED AT A STUDENT AFFECTED BY DUCHENNE MUSCULAR DYSTROPHY (DMD)}

\author{
José A. Benavent* \\ Julián García Blanco** \\ Universitat de València
}

\section{RESUMEN}

El desarrollo de la LOGSE (1990) sobre la atención a la diversidad en los Institutos de Educación Secundaria (IES) implica una sensibilización y una disponibilidad para el cambio cultural del quehacer diario, como la organización y gestión del centro, la adaptación del Proyecto curricular, la eliminación de las barreras arquitectónicas, la asignación de los recursos físicos, la coordinación entre los distintos departamentos didácticos y el Departamento de Orientación con la meta de ofrecer una atención adecuada y eficiente al alumnado y a sus familias.

En este artículo pretendemos exponer los procesos aplicados tanto en el asesoramiento psicopedagógico (Benavent, 1999) como en la orientación vocacional (García y Ferrer, 1997) en un alumno con Distrofia Muscular Duchenne (DMD) que ha cursado los tres cursos de BUP y en la actualidad está matriculado en COU.

Palabras clave: Asesoramiento psicopedagógico, orientación vocacional, Distrofia Muscular Duchenne.

\section{ABSTRACT}

The LOGSE (1990) development to improve diversity in education at the «Institutos de Educación Secundaria» (IES) requires sensitivity and availability of cultural change, center's organization and

* José Antonio Benavent Oltra. Profesor titular del Departamento de Didáctica y Organización Escolar de la Universidad de Valencia. Líneas de investigación: Historia de la Orientación y la Psicopedagogía en España, metodología de la toma de decisiones al finalizar la ESO, y análisis, evaluación y prospectiva del modelo institucional de Orientación educativa, psicopedagógica y profesional en la actual Reforma educativa.

** Julián García Blanco. Jefe del Departamento de Orientación del Instituto de Enseñanza Secundaria «Quart» de Quart de Poblet (Valencia). Líneas de investigación: Metodología de la toma de decisiones al finalizar la ESO y Estrategias para el autoaprendizaje. 
management, curriculum adaptation, removal of architectural barriers, special equipment, coordination among all didactic departments and guidance department, etc. All these changes in order to offer a quality education for the students and their families.

This article presents the guidance process (Benavent, 1999) and vocational counselling (García y Ferrer, 1997) aimed at a student with Duchenne muscular dystrophy (DMD), who passed three courses of BUP and at present is enroled in COU (the last year of high school).

Key words: High school guidance, vocational counseling, Duchenne muscular dystrophy (DMD).

\section{Presentación}

La aplicación progresiva de la LOGSE (1990), desde la perspectiva de la atención a la diversidad, implica la matriculación de alumnos con diferentes necesidades educativas permanentes en los Institutos de Educación Secundaria (IES), lo que supone un cambio fundamental en la dinámica ordinaria de los centros educativos, ya que con la LGE (1970) eran los claustros los que solicitaban a la Administración educativa que se les nominara como Centro de integración.

El principio de igualdad de oportunidades en educación implica que para que las desigualdades y desventajas sociales o culturales de las que determinados alumnos parten no acaben convirtiéndose en desigualdades educativas, se pongan en marcha una serie de medidas positivas de carácter compensador (RD 299/1996).

La matriculación en un IES de un alumno con Distrofia Muscular Duchenne (DMD), dadas las peculiaridades que implica el cuadro clínico, representa un reto para todos los profesionales del centro escolar, incluyendo al Departamento de Orientación. Plantear un trabajo de atención psicopedagógica en un caso cuyo pronóstico a no muy largo plazo es la muerte (esperanza de vida que oscila entre los 15-25 años), supone abordar un campo de trabajo novedoso no sólo para nuestro departamento, sino posiblemente para la mayor parte de centros de enseñanzas medias en que este hecho pudiera darse.

Una dificultad añadida con la que nos hemos encontrado ha sido la escasez de referencias bibliográficas que plasmen el proceso seguido con estos alumnos, que aludan al asesoramiento vocacional, no sólo para la $\mathrm{DMD}$, sino para cualquier proceso degenerativo que afecta a la edad escolar. Así, si la documentación científica referida al asesoramiento vocacional es limitada en el área de las necesidades educativas especiales, la característica antes citada de la DMD, justifica el poco desarrollo de la literatura específica.

Nuestro propósito es dejar constancia del proceso seguido desde el Departamento de Orientación con la identificación de la situación en la que se encontraba al incorporarse a nuestro IES, a sus 14 años, facilitarle el asesoramiento y orientación adecuada a su realidad durante todo el tránsito por los tres cursos de BUP y el actual en COU y potenciar sus estrategias y/o capacidades para lograr el éxito en su proceso de aprendizaje y su futura decisión vocacional. 


\section{Marco Teórico}

\section{El Síndrome de Duchenne}

La Distrofia Muscular Duchenne o Síndrome de Duchenne es una de las diversas formas de miopatías degenerativas que existen, y que suelen afectar aproximadamente entre 46 niños de cada millón que nacen. Es, de entre todas las distrofias musculares, la más maligna pues los hombres que la padecen en raras ocasiones pueden llegar a la edad adulta, planteando muchas dudas a la hora de su escolarización y, por ende, al trabajo de su orientación vocacional. Para abordar esta dificultad es necesario que el profesional encargado de esta orientación y asesoramiento conozca las dificultades que plantea esta enfermedad y cómo puede ofrecer la máxima calidad de vida posible (García y Ferrer, 1997).

La DMD es hereditaria, se trasmite de forma recesiva ligada al cromosoma $\mathrm{X}$, por lo que sólo lo padecen los hombres, aunque es trasmitida por las mujeres. Se caracteriza por una progresiva degradación de la masa muscular que se sustituye en ocasiones por tejido adiposo. Las primeras manifestaciones externas suelen aparecer en torno a los 4 años de edad, mostrando el niño dificultades crecientes y torpeza a la hora de subir y bajar escaleras, o cuanto intenta ponerse de pie. Su forma de caminar comienza a ser patosa mostrando una especie de balanceo. A partir de entonces cada caso sigue un ritmo peculiar de pérdida de fuerza muscular y de capacidad de movimiento; pronto será necesario la utilización de la silla de ruedas y en unos años se acabará en un estado de inmovilización total, necesitando a veces respiración asistida. En sus inicios muestran un engañoso aspecto musculoso por hipertrofia, lo que aparentemente puede enmascarar su tremenda debilidad muscular; igualmente se atrofian los músculos faciales apareciendo una expresión característica en su cara (faces miopática).

En la actualidad es posible detectar la presencia del síndrome previo al nacimiento mediante análisis genético. También podemos conocer con bastante fiabilidad si una mujer es portadora. Por contra, no se conoce ningún tratamiento que pueda detener o paralizar el proceso, aunque la esperanza de vida últimamente se ha conseguido alargar en algunos años gracias a la mejora de los cuidados paliativos.

De la escasa bibliografía encontrada, hay un estudio hecho en Irlanda y Estados Unidos (CAROL FizPATRICK y CiARAN BARRY, 1990) comparando grupos de familias en las que alguno de sus miembros padecía la enfermedad. Se pudo comprobar la escasez de comunicación que existía entre los miembros de la familia y la tensión que sentían incluso al pedir ayuda a grupos de asesoramiento. Appleton, Bushby, Gardner, Welch y Kelly (1991) intentaron, por su parte, relacionar la macrocefalia que sufren algunos afectados con retraso mental, no pudiéndose establecer una correlación significativa entre ambos hechos. En un estudio sobre ajuste social y psicopedagógico realizado en el Hospital «La Fe» de Valencia (RoSelló, Soriano, Miranda, Mulas, Barbero y Moreno, 1995) se concluye que es el retraso evolutivo acentuado en el área motora, el que provoca el retraso en el aprendizaje.

También se ha investigado la experiencia emocional en la conducta facial, la mirada y las interacciones diádicas, concretamente en el uso de estructuras verbales (el tiempo futuro, frases en infinitivo, el tiempo presente y el imperativo) (MERTEN, 1997), la respuesta emocional de la expresión facial ante fotografías como estímulo (CARROLL y RUSSELL, 1997), la reacción emocional y la sonrisa en sujetos con SDM y no-SDM (SURAKKA y HIE- 
TANEN, 1998), la sonrisa en la interacción con los padres (DICKSON, WALKER y FogEL, 1997), la correlación entre risa y sonrisa en momentos de aflicción como la muerte de un pariente (KELTNER y BONANNO, 1997) y las características lingüísticas y comunicativas de familias con hijos con DMD y sin-DMD (BERNicot, CHAigneau y cols., 1997).

En conclusión, hemos de destacar que los pocos estudios realizados, se han centrado en comprobar un retraso mental en los niños con DMD, y han abordado los efectos emocionales que para la familia tiene la aparición de un diagnóstico con un pronóstico tan devastador. Con respecto al primer punto cabe comentar que se ha confundido en muchas ocasiones un bajo rendimiento académico, debido a las crecientes dificultades en la movilidad que van apareciendo y progresando, y la pérdida de motivación e interés escolar unida a los problemas emocionales con una pobreza intelectual. Se intentaba justificar un «retraso mental», constatando que en absoluto se puede sustentar desde una posición organicista dado que este tipo de distrofia muscular no afecta al tejido neuronal que soporta nuestras funciones superiores. Por otro lado, los estudios referidos a las consecuencias familiares reflejan graves disfunciones de la comunicación en el seno de la familia, apareciendo en muchos casos temas tabú que también pudimos constatar en nuestro caso estudiado: la propia enfermedad, la muerte, referencias con perspectiva de futuro...

\section{Marco teórico de referencia para la orientación vocacional}

La conducta vocacional es un complejo proceso de socialización que cada persona va configurándose en un medio sociocultural determinado y que implica la relación dialéctica de los dos elementos que la configuran: la satisfacción de las necesidades de desarrollo individual y la adecuada cobertura de las necesidades sociales. Así pues, se trata de la resultante entre los procesos de sociogénesis (familia, mundo laboral, sistema educativo y factores situacionales) y psicogénesis (biodatos, género y pertenencia a minorías, intereses, personalidad, aptitudes y toma de decisiones) (Rivas, 1998). En este trabajo en concreto nos referimos a una persona que pertenece a minorías entendiendo por tales, aquéllas que tienen características particulares y que, en función de ello, son sujetos con necesidades educativas especiales generalmente originadas por déficits físicos y/o perceptivos. En este caso nos encontramos ante un déficit fundamentalmente físico.

Puesto que la sociedad opta por la integración de las diferencias, esta integración debe prolongarse al mundo laboral. A partir de la acción educativa especial ajustada a sus peculiaridades, procede tratar de la adaptación de su conducta vocacional en el mayor número de áreas ocupacionales que sea posible, intentando aunar tanto nuestra intervención técnica como la incorporación de nuevas ayudas tecnológicas que hacen posibles ciertas realidades que hasta hace poco tiempo parecían inalcanzables.

De este modo, entendiendo la conducta vocacional como parte del proceso de socialización, y ante la libertad para elegir, pueden producirse situaciones en que, la persona en función de su maduración personal, importancia de las opciones y de la situación del entorno, puede necesitar algún tipo de ayuda que le oriente en su adecuación al mundo productivo. El asesoramiento vocacional es la forma técnica de ofrecer y prestar esa ayuda. En este sentido, nuestro trabajo ha intentado analizar algunos de estos aspectos, centrándose fundamentalmente en la situación de madurez personal del alumno, y en la situación de su entorno. El análisis de las opciones, dada la edad y la heterogeneidad de la evolución de la situa- 
ción física de los efectos del DMD, es un punto, en nuestra opinión, a postergar dentro del proceso de asesoramiento vocacional del caso estudiado.

\section{METODOLOGÍA}

Se procedió, a través de entrevistas, a recopilar la documentación familiar correspondiente al diagnóstico neurológico y historia clínica. Se contactó con el Departamento de Orientación del centro de EGB para que facilitara su historial académico. Se aplicó una exploración inicial a todos los alumnos de Primero de BUP, entrevistas con los Equipos Docentes (primero para facilitarle la información básica de las características del alumno y posteriormente para analizar su proceso de aprendizaje).

Igualmente se han mantenido sesiones de trabajo con el alumno para facilitarle la toma de decisiones en cuanto a elección de EATP $\left(2^{\circ}\right.$ y $3^{\circ}$ de BUP $)$, opción de COU, utilizando la técnica del portafolios (IBARRA y otros, 1999).

Dada la singularidad del caso objeto del trabajo en cuanto a la evaluación de su motricidad fina y la escritura, se optó por la investigación idiográfica, que es un estudio de los fenómenos basado en su irrepetibilidad. El diseño de caso único utilizado ha constituido un criterio cambiante; es decir, la conducta del sujeto cambia, con lo cual la exploración debe ir adaptándose al ritmo de evolución del sujeto. En síntesis, el Diseño (HARTMAN Y HALL, 1976) consiste en determinar la Línea Base, que es seguida de una intervención hasta conseguir el criterio preestablecido; entonces, éste se convierte en la nueva Línea Base y se establece un nuevo criterio hasta obtener el criterio final.

El inicio, se diseñó trabajar directamente con el alumno en cinco ámbitos:

a) Estado de salud.

b) Ámbito familiar y social.

c) Proceso enseñanza - aprendizaje.

d) Intereses profesionales.

e) Institución escolar.

Para identificar su nivel actual en las Habilidades básicas de Inteligencia, se tuvieron en cuenta los resultados del BADYG-M- (CARLOS YUSTE, 1990) y para la escritura se planificó una copia con bolígrafo y otra con ordenador, más una descripción escrita. Por último, se exploraron sus áreas de interés vocacional a través del Cuestionario de Intereses Vocacionales de MARTínez y Rivas (1988).

\section{Presentación del caso}

Varón, nacido en junio de 1982. Es el mayor de dos hermanos. Matriculado el curso 1996-97 en $1^{\circ}$ de BUP en un Instituto de la Comunidad Valenciana. 


\section{a) Estado de salud}

Desde su matriculación en el IES (GARCíA Y FERRER, 1997) ha evolucionado lentamente con un deterioro progresivo, que le exige utilizar de forma continua la silla de ruedas, ya no le permite la enfermedad mantenerse en bipedestación, le ha aumentado su desviación de columna (lordosis), incluso en la actualidad tiene molestias de cadera. Su fatiga se acentúa por las tardes, especialmente, en aquéllas que tiene clases. Todavía se mantiene autónomo en los hábitos de la vida diaria como la comida, aunque para otros necesita asistencia (aseo y vestido).

Hace tres años se le aconsejó por parte de la médico especialista que se le cambiara la silla de ruedas con el respaldo más alto y sujeción de hombros, recomendación realizada, y que utilizara una silla de ruedas eléctrica para sus desplazamientos; esta última pauta médica todavía no se ha puesto en práctica ya que él no quiere cortar la relación con sus compañeros, encargados voluntariamente, de trasladarlo desde casa al IES y dentro del Instituto.

Continua siendo una persona reservada, cohibida. En general los rasgos descritos en nuestro anterior trabajo se mantienen, posiblemente con una mayor centración en sí mismo. Valora positivamente su estancia en el Instituto.

\section{b) Ámbito familiar y social}

Destacar la importancia que tiene la forma de transmitir el diagnóstico a los padres para su futura actitud y satisfacción personal (GREEN y MURTON, 1996). Lo que distinguía a los padres de hijos con DMD, por ejemplo, del Síndrome de Down, era que los doctores les habían comunicado a los primeros una sentencia de muerte para su niño. Este trabajo muestra que el diagnóstico puede ser dado de una forma satisfactoria para los padres. Para ello, se requiere empatía y sensibilidad que atienda las necesidades emocionales e informativas de los padres.

El apoyo de la familia es constante, ayudándole tanto en las actividades escolares, como en su tiempo de ocio. A través de una asociación de padres, los fines de semana práctica el hockey en sillas de ruedas con la colaboración de unos cuantos voluntarios. La asociación de padres organiza, también, salidas a otras ciudades, concretamente para este curso tienen programado un partido de hockey en Barcelona.

Mientras que en la EGB sus compañeros de clase lo incluían en sus paseos, incluso en la asistencia a alguna discoteca, al pasar los años e ir haciéndose adolescentes y empezar a salir con chicas, él se ha visto relegado a su entorno familiar; aspecto que en alguna ocasión ha lamentado y aceptado, aunque con cierta resignación.

\section{c) Proceso enseñanza-aprendizaje}

Asiste a clase de forma continua. Podemos considerar su evolución educativa normal, según recogemos en la siguiente tabla: 


\begin{tabular}{|l|c|c|c|c|}
\hline & Curso 1996-97 & Curso 1997-98 & Curso 1998-99 & Curso 1999-00 \\
\hline Nivel & $1^{\circ}$ BUP & $2^{\circ}$ BUP & $3^{\circ}$ BUP & COU \\
\hline Exención & Ed. Física & Ed. Física & Ed. Física & \\
\hline Rendimiento & Aprobado en Junio & Aprobado en Junio & Una pendiente & \\
\hline Nota media & 5.94 & 7.06 & & \\
\hline
\end{tabular}

Se valora como capacitado para aprobar COU. Su rendimiento ha sido normal, y su esfuerzo bueno. Muy posiblemente su método de trabajo personal no ha evolucionado, ya que se sigue caracterizando por realizar diariamente las actividades solicitadas por el profesorado y unos días antes de los exámenes preparárselos. Por tanto podemos afirmar que su estilo de aprendizaje es memorístico, y muy semejante al que utilizan bastantes compañeros de su aula.

Consideramos que el hecho de ser consciente de su salud le condiciona notablemente las decisiones a tomar día a día; no olvidemos que valora el Instituto como una obligación. Esta actitud vital le lleva a plantearse objetivos a muy corto plazo, aspecto fundamental para comprender su futuro profesional.

\section{d) Intereses profesionales}

En el ámbito personal, en cuanto a sus expectativas a corto y largo plazo responde que «no lo he pensado». Esta circunstancia que, por una parte es común a un porcentaje elevado de adolescentes de esta edad, puede también obedecer a sus expectativas o actitud vital hacia su enfermedad, cuyo pronóstico y consecuencias son temas que en todo momento, tanto la madre como el mismo alumno, evitan comentar. Completamos esta parte en el siguiente apartado de Intereses Vocacionales.

\section{e) Ámbito Institucional}

La opinión mayoritaria del profesorado y de los tutores se caracteriza por considerar su integración en el Instituto y su conducta como buenas. Señalan que hay que planificar con antelación su participación en las actividades, ya que necesita más espacio, e igualmente hay que buscar una mesa adaptada para que pueda escribir. Lo anterior obligó al Centro a reestructurar la distribución de las aulas, y asignarle a su grupo un aula que se encuentra en la planta baja.

Algún profesor se planteó dudas acerca de qué podía exigirle y qué no debía pedirle; incluso se interrogaban acerca de si es conveniente preguntarle en clase, o ignorarlo para que el grupo no se fijara más en él, por los problemas que a veces mostraba en su voz (microfonía asociada a la pérdida de fuerza muscular en cuerdas vocales), acentuada por la ansiedad de la situación.

Un aspecto que nuestro Instituto sí cumplía era la disposición de rampas de acceso al edificio y de un ascensor para subir al laboratorio y aula de dibujo que se encuentran en el primer y segundo piso, respectivamente. La labor de traslado por los diferentes pisos 
ha sido realizada con generosidad tanto por los compañeros como por los conserjes del Instituto.

\section{Datos de las exploraciones psicopedagógicas realizadas}

\section{Aptitudes Intelectuales}

Un estudio de Cotton, Crowe y Voudouris, (1998) demostró que los sujetos con DMD obtuvieron peor funcionamiento en tests de atención compleja, fluidez verbal y funciones de memoria no verbal, sugiriendo que el déficit cognitivo en la población DMD puede ser de naturaleza específica más que global.

TRIFILETTI y otros (1994) analizaron la existencia del retraso mental en 53 pacientes italianos con distrofia muscular. El retraso mental fue detectado en 9 de 40 pacientes con DMD (representa un $22.50 \%$ de la muestra). Éstos datos apoyan la creencia de que las diferencias en la frecuencia del retraso mental Duchenne MD y Becker MD tienen una base genética. Por otra parte, Bushby, Appleton, ANDERson y Welch (1995) afirman que el déficit intelectual observado en sujetos con DMD puede ser la consecuencia de una hipoxia cerebral

A nuestro alumno se le aplicó el BADYG-M de Carlos Yuste Hernández (1990) en Diciembre de 1995, cuando cursaba $8^{\circ}$ de EGB. En madurez intelectual general obtiene una PC de 60; en Inteligencia General Verbal, PC 66; en inteligencia general no verbal, PC 54; en memoria, PC 77 y en percepción de diferencias, PC 19.

Contrasta el resultado obtenido con los estudios analizados por P. Dague (en Ajuriaguerra, 1979) que, en un estudio con 103 chicos, afirma que el $40 \%$ de los sujetos tienen un CI inferior a 80 , y el $22 \%$ un CI inferior a 70 .

\section{Habilidades Básicas}

\section{Lectura}

Billard, C., y otros (1998) analizaron las habilidades lectoras de 21 niños (edad 8.6 13.6 años) con DMD, de 11 niños (edad 8.3 - 13.6 años) con atrofia muscular espinal (SMA) y 42 niños (edad 6.2 - 12.3 años) que recibieron educación normal. Los datos indican que los sujetos con DMD manifestaban una edad lectora que era significativamente más baja que los sujetos con SMA comparados con su edad cronológica. Estas dificultades de aprendizaje no fueron relacionadas con un déficit en la ejecución de la inteligencia no verbal, pero la evaluación psicolingüística mostraba un déficit en la inteligencia verbal, especialmente en los subtest de Semejanzas y Aritmética del WISC-R, en las habilidades fonológicas, en la repetición de la palabra oral y en dígitos. Los resultados de los sujetos de DMD eran heterogéneos, y con un amplio rango de compromiso (grande, normal o pequeño). Para clarificar la naturaleza de esta deficiencia lectora en los sujetos DMD, los 3 grupos fueron evaluados a través de la lectura de una lista de palabras simples y sin sentido. Los rendimientos de los niños y adolescentes DMD eran significativamente más bajos en palabras sin sentido, sugiriendo una disfunción similar a la dislexia disfonética. 
En la prueba aplicada en noviembre de 1996 se obtuvieron los siguientes resultados:

\begin{tabular}{|l|c|c|c|}
\hline \multicolumn{1}{|c|}{ C/1999-97 } & Rendimiento & Media del aula & DS \\
\hline Velocidad Lectora & $194 \mathrm{pxm}$ & 173.44 & 33.92 \\
\hline Comprensión lectora & 1 & 2.99 & 1.39 \\
\hline Eficiencia lectora & 2 & 3.19 & 1.02 \\
\hline
\end{tabular}

Tiene una velocidad superior a la de su grupo de referencia, si bien, ello no contribuye en absoluto a la comprensión y por ende a la eficiencia en vista de los bajos resultados obtenidos en estos apartados (Escala de 0 a 10).

\section{Escritura}

Dado el déficit motor que implica su enfermedad, se diseñó una evaluación inicial para identificar el estado actual de sus alteraciones en la motricidad fina y las repercusiones que en el ámbito de la escritura acarrean.

\begin{tabular}{|c|c|c|c|}
\hline C/1996-97 & Palabras texto & Tiempo dedicado & Palabras por minuto \\
\hline Bolígrafo & 54 & $135 ”$ & 24 \\
\hline Ordenador & 48 & $269 "$ & 10.71 \\
\hline
\end{tabular}

La escritura caligráfica es, por el momento, totalmente legible y los datos muestran una rapidez, a falta de práctica en el ordenador y de un mayor conocimiento de la disposición de las letras en el teclado, bastante superior en favor de la escritura manual. Así, no se contempla todavía el uso del ordenador como ayuda técnica, si bien, será necesario vigilar la evolución para valorar el momento adecuado de su introducción pues en el futuro será un elemento esencial, junto con otro tipo de adaptaciones para facilitar sus movimientos sobre el teclado.

Durante el presente curso 1999-2000, con el objetivo de verificar su estado actual y de cumplimentar el protocolo exigible por la Universidad para solicitar una ampliación de tiempo en la Prueba de Acceso a la Universidad (PAAU), según recoge la normativa de la Universitat de Valencia aprobada el 30 de abril de 1998, se ha procedido a realizarle otra prueba de escritura (Enero 2000), obteniendo el siguiente resultado:

\begin{tabular}{|c|c|c|}
\hline Texto de $\mathbf{1 0 0}$ palabras & Duración & Referencia del grupo normativo \\
\hline Escritura manual & 310 segundos & 252 segundos \\
\hline
\end{tabular}

Al facilitar la Asesoría Universitaria para los estudiantes con discapacitación de la Universitat de Valencia (Guía curso 1999-2000) el texto y el baremo, constatamos que la pérdida de rapidez en la escritura manual ha sido aproximadamente de un $24 \%$ en estos tres años. Por tanto hemos solicitado una adaptación temporal de las distintas pruebas. 


\section{Expresión escrita libre: Descripción del centro}

(27 febrero 1997). Se solicitó una redacción con una duración de 15 minutos, tomando control a los 7'30' y a los 15 '.

\begin{tabular}{|l|c|c|c|}
\hline & $\mathbf{1}^{\text {a }}$ Parte & $\mathbf{2}^{\text {a }}$ Parte & Total \\
\hline Rendimiento & 136 & 70 & 206 \\
\hline Media del aula & 123.59 & 102.50 & 223.15 \\
\hline D.S. del aula & 30.12 & 33.55 & 58.86 \\
\hline
\end{tabular}

Mientras que en la primera parte su rendimiento es superior a la media de su clase, en la segunda baja su rendimiento, aunque no de forma significativa, en referencia al rendimiento de su grupo. Tomando como punto de análisis el rendimiento personal del alumno, el descenso en el nivel de producción de los primeros siete minutos y medio a los segundos, resulta bastante significativo $\left(X^{2}=21,14, \propto=0,001\right)$. Obvia decir que por lo general, en una prueba abierta con estos rasgos y en estas edades, el descenso de producción en relación con el tiempo es muy característico, si bien, el desequilibrio es muchísimo más evidente en nuestro alumno (obsérvese la media final de producción). Con estos resultados cabría plantearse si dicha disminución se debía a una pobreza de ideas o a su baja tolerancia a la fatiga, síntoma evidente de su alteración. Para confirmar esta hipótesis se realizó una prueba de copia, libre de influencia en cuanto a producción de ideas, obteniéndose estos resultados:

\begin{tabular}{|l|c|c|c|}
\hline & $\mathbf{1}^{\text {a }}$ Parte & $\mathbf{2}^{\text {a }}$ Parte & Total \\
\hline Rendimiento & 183 & 174 & 357 \\
\hline
\end{tabular}

En esta prueba se observa que la diferencia entre ambas partes no es significativa estadísticamente, lo que nos permite afirmar por una parte que, en la medida que aumenta el tiempo de trabajo disminuye ligeramente la producción escrita y, en segundo lugar, la causa de esta disminución productiva se debería en este caso a la pobreza de ideas, y no tanto a la fatiga física.

\section{Personalidad}

SuZuKi, K. (1995) estudia el cambio de actitudes, durante un período de 18 a 24 meses, de 5 pacientes varones (edad 16 a 27 años) con DMD hacia su progresivo debilitamiento, como enfermedad terminal. Los resultados indican que los pacientes siempre sienten "ansiedad ante la muerte» y que el grado de esta ansiedad estaba exactamente relacionado con el estado progresivo de DMD, identificando los siguientes seis niveles:

I. Conocimiento incierto de la ansiedad de la muerte

II. Represión de la ansiedad de la muerte y corto período de estabilidad

III. Conocimiento conciso de tener la ansiedad de la muerte 
IV. Evitación de la ansiedad de la muerte

V. Confrontación al miedo de la muerte

VI. Aceptación de la inevitabilidad de la muerte

Para el autor hay dos niveles que requieren mayor soporte psicológico: el III y V; en el estadio III la ansiedad de la muerte es probable llegue a ser concisa, produciendo angustia y confusión; y en el estadio V, como la DMD está marcadamente agravada, la ansiedad de la muerte llega a ser una inevitable consecuencia de que debe ser confrontada. La evidencia sugiere que la buena comunicación entre el alumno y los apoyos psicológicos es extremadamente importante para que éste hábilmente confronten y acepten la naturaleza progresiva y terminal de la DMD.

En diciembre de 1995 se le aplicó el HSPQ, de CATtell (1983). Atendiendo a lo más destacable, puntúa significativamente en los rasgos de Sobrio-Entusiasta (decatipo 3); Cohibido-Emprendedor (decatipo 3). Es de destacar que una de las características de personalidad que se detecta en la poca documentación científica existente sobre la DMD es que estos sujetos suelen ser retraídos. Tiene una clara tendencia a la introversión como podemos deducir de las entrevistas personales, afirmaciones de la familia y comentarios del profesorado. Si bien, se constata una discrepancia entre los rasgos de personalidad manifestados en casa, donde no es tan retraído e incluso se muestra en ocasiones agresivo, y los del centro; donde la deseabilidad social condiciona su conducta.

\section{Intereses Vocacionales}

En diciembre de 1995 se pasó el Cuestionario de Preferencias Profesionales de CARLos Yuste (1988), destacando las siguientes preferencias: Artes Plásticas (PC 99), Hostelería (PC 99), Técnico Industrial (PC 96) y Artes Musicales (PC 94)

Durante el curso 1996-97 se le pasó en abril el Cuestionario de Intereses Vocacionales, subtest del SAV (RIVAS et al., 1988), obteniendo las siguientes Áreas Vocacionales en primer lugar: Teórico-Humanística, Administrativo-Burócrata, Aire Libre-Deportiva con una puntuación de 13 y en cuarto lugar el área Persuasivo-Comercial con 12 puntos. Las cuatro puntuaciones en el baremo corresponden a una calificación de 4.

En primer lugar hemos de constatar la evolución de intereses profesionales de un curso al siguiente, hecho normal en alumnos adolescentes. En segundo lugar, nos llama la atención que elija en primer lugar el área vocacional de «Aire Libre-Deportiva» cuando su estado de salud le impediría ejercer profesiones de este ámbito, aunque nos consta su gran afición por los deportes y especialmente el fútbol.

En $2^{\circ}$ y $3^{\circ}$ de BUP se matriculó en la EATP de Informática.

La opción elegida en COU, curso 1999-2000 ha sido la relacionada con las carreras universitarias de Letras. En la entrevista personal se mostraba un tanto escéptico en cuanto a tomar decisiones de futuro a largo plazo (elegir una carrera o profesión para él lo ve «un poco cuesta arriba», «no lo tengo claro»), aunque sí muestra cierta simpatía por la informática, pero le desanima no poder alcanzar la línea de corte que le exija la Prueba de Acceso a la Universidad (PAAU). 


\section{Consideraciones}

- La incorporación de un alumno a un IES con las características especificadas, distrofia muscular Duchenne implica las modificaciones en la gestión de los recursos físicos del centro (adecuación de aula, eliminación de barreras arquitectónicas, uso de ascensores, ...) y la colaboración de todos los recursos humanos (equipo directivo, equipos docentes, departamento de orientación, conserjes, ...) disponibles en el centro.

- Consideramos necesario facilitar la información básica a los Equipos Docentes acerca de la enfermedad, para que quede claro y sepan diferenciar aquello que se produce por causa de la misma y lo que puede ser considerado como normal a todo alumno de esa edad y, a la vez, les capacite para un tratamiento didáctico y humano adecuado.

- Su proceso de enseñanza-aprendizaje, desde la evaluación inicial, nos planteó la necesidad de trabajar las aptitudes básicas con el alumno, especialmente la aptitud espacial. Potenciar la comprensión lectora y, especialmente, la flexibilización de sus estrategias de aprendizaje.

- Dadas sus características personales, se le ofertó el entrenamiento en habilidades sociales, y la intervención en los aspectos de introversión, fomentando en el aula la participación a través del trabajo cooperativo.

- La evolución de su enfermedad nos ha conducido a mantener el seguimiento de sus habilidades básicas en escritura tomando como referencia los resultados obtenidos en el $2^{\circ}$ trimestre de Primero de BUP, para ver si se produce algún deterioro. Consideramos que necesitará en un futuro próximo el uso del ordenador por la pérdida de motricidad fina para realizar el trazo gráfico.

- En cuanto a los intereses vocacionales se ha observado la necesidad de un apoyo individualizado, por parte del orientador donde se ha trabajado el ajuste de expectativas, entre sus aspiraciones legítimas y sus limitaciones reales. Su proceso vocacional se caracteriza por un estado de indefinición y con cambios bruscos (contraste entre los resultados de $8^{\circ}$ de EGB y $1^{\circ}$ de BUP), por otro lado propios de su edad. Sin embargo, este aspecto se ha continuado trabajando en el asesoramiento del itinerario académico, por ejemplo en la elección de EATP en BUP (informática) y opción en COU (Letras). Ahora bien, esta labor de mediación siempre se ha supeditado a las características personales y específicas del alumno.

- Al finalizar el curso de segundo de BUP, dada la evolución de su enfermedad y la colaboración directa de algunos compañeros, se consideró necesario la solicitud de un educador en el centro para cubrir necesidades de desplazamiento, avance y alimentación. Constatamos que esta solicitud se realizó en Junio de 1998 y la Administración todavía no ha contestado.

- Se ha trabajado de forma continua en coordinación con la familia, ofreciéndole el psicopedagogo la ayuda y asesoramiento sobre la evolución de su hijo, y atendiendo las demandas que nos han planteado, siendo la principal fuente de información sobre la evolución real de la enfermedad. 


\section{Referencias bibliográficas}

Ajuriaguerra, J. de (1979): Manual de psiquiatría infantil. Barcelona: Editorial Toray-Massón.

Appleton et al. (1991): Head circumference and intellectual performance of patients with duchenne muscular dystrophy. En Developmental Medicine and Child Neurology, 33: 884-890.

Benavent, J. A. (1999) La orientación psicopedagógica en el umbral del s. XXI: Una mirada al futuro. En AEOP, 17: 53-62.

Bernicot, J., Chaigneau, A., Col, G. (1997): L'expression du futur chez des patients atteints de dystrophie musculaire de Duchenne: Une etude de cas en milieu familial. En Approche Neuropsychologique des Apprentissages chez l'Enfant. 9 (1): 26-33.

Billard, C., y otros (1998): Reading ability and processing in Duchenne muscular dystrophy and spinal muscular atrophy. En Developmental Medicine and Child Neurology. 40 (1): 12-20.

Bushby, K. M., Appleton, R, Anderson, L., Welch, J. L. (1995): Deletion status and intelectual impairment in Duchenne muscular dystrophy. En Developmental Medicine and Child Neurology. 37 (3): 260-269.

Carroll, J. M. y Russell, J. A. (1997): Facial expressions in Hollywood's protrayal of emotion. En Journal of Personality and Social Psychology. 72 (1): 164-176.

Cotton, S., Crowe, S. F., Voudouris, N. (1998): Neuropsychological profile of Duchenne Muscular Dystrophy. En Child Neuropsychology. 4 (2): 110-117

Dickson, K. L., Walker, H., Fogel, A. (1997): The relationship between smile type and play type during parent-infant play. En Developmental Psychology. 33 (6): 925-933.

Ferrer Manchón, A. M. (1996): El desarrollo de los niños con déficits motores. En Fortes, $M^{\mathrm{a}}$ Carmen, Ferrer, Antonio y Gil, Ma Dolores (Coord) Bases Psicológicas de la Educación Especial: Aspectos teóricos y prácticos. Valencia: Ed Promolibro.

Sher, R. A. (1950): Statistical Methods for Research Workers. Nueva York: Hafner Publishing.

Fizpatrick, C. y Barry, C. (1990): Cultural differences in family communication about duchenne muscular dystrophy. En Developmental Medicine and Child Neurology, 32: 967-973.

García, J. y Ferrer, A. (1997): Asesoramiento Vocacional con un caso de distrofia muscular tipo «Duchenne», en Alcantud, F. (Editor). En Universidad y Diversidad. Valencia, pp: 75-90.

L. J. y Albarrán, A. (1983): La distrofia muscular. Madrid: Ed Popular.

Green, J. M. y Murton, F. E. (1996): Diagnosis of Duchenne muscular dystrophy: Parents' experiences and satisfaction. En Child: Care, Health and Development. 22 (2): 113-128.

Guia de la Universitat de València (1999-2000). València: Vicerectorat dÉstudiants.

Hartmann, D. P. y Hall, R. V. (1976): A discusion of the changing criterion design. (En Journal of Applied Bechavior Analysis, 9: 527-532.

Ibarra, M. y Sánchez, C. (1999): Posibilidades del portafolios en auto-orientación profesional. En REOP, 17: 63-82.

Keltner, D., Bonanno, G. A. (1997): A study of laughter and dissociation: Distinct correlates of laughter and smiling during bereavement. En Journal of Personality and Social Psychology. 73 (4): 687-702.

Merten, J. (1997): Facial-affective behavior, mutual gaze, and emotional experience in dyadic interactions... En Journal of Nonverbal Behavior. 21 (3): 179-210.

Rivas Martínez, F. (1988) Sistema de autoayuda Vocacional. Valencia: Generalitat Valenciana.

Rivas Martínez, F. (1998): Manual del SAV-R y SAVI-2000. En Sistemas de Autoayuda y Asesoramiento Vocacional. Valencia: Servicios de Asesoramiento Vocacional y Educativo. 
Bert Berkow, M. D. (Ed) (1989): El Manual Merck de Diagnóstico y terapeútica. (8a edición). Ediciones Doyma.

Roselló, M. B. et al. (1995): Ajuste psicosocial y psicopedagógico. de los niños con distrofia muscular progresiva de Duchenne. Comunicación presentada el 17-6-1995 en la XII Reunión anual de la Sociedad Valenciana de Pediatría. En Boletín de la Sociedad Valenciana de Pediatría, ${ }^{\circ} 15$ : 161-162.

Surakka, V. y Hietanen, J. K. (1998): Facial and emotional reactions to Duchenne and non-Duchenne smiles. En International Journal Of Psychophysiology. 29 (1): 23-33.

Suzuki, K. (1995): The psychological world of patients with Duchenne Muscular Dystrophy: Attitude toward the illness and death. En Japanese Journal of Child and Adolescent Psychiatry. 36 (4): 271-284.

Trifiletti, R. R. y otros (1994): Mental retardation in muscular dystrophy: A study from Catania, Italy, with a review of the literature. Developmental Brain Dysfunction. 7 (4-5): 183-191.

\section{Normativa específica}

LOGSE 1/1990 de 3 octubre, Ley Orgánica de Ordenación General del Sistema Educativo (BOE, 4 octubre).

LEY 14/1970 de 4 agosto, General de Educación y financiamiento de la Reforma Educativa (BOE, 6 de agosto).

RD 299/1996 de 28 febrero, Real Decreto de Ordenación de las acciones dirigidas a la compensación de desigualdades en educación (BOE, 12 marzo).

Fecha de recepción: 15-3-00

Fecha de revisión: $25-4-00$

Fecha de aceptación: 21-11-00 